\title{
New Developments in Energy-filtering Transmission Electron Microscopy
}

\author{
F. Hofer, B. Schaffer, W. Grogger and G. Kothleitner \\ Research Institute for Electron Microscopy, Graz University of Technology, Steyrergasse 17, \\ A 8010 Graz, Austria
}

With the spreading of micro- and nanotechnology there will be an increasingly strong need for imaging techniques that allow high spatial resolution studies of nanodevices and nanostructured materials. Here energy-filtering transmission electron microscopy (EFTEM) plays a central role as a means of measuring chemical and structural properties of very small regions of a thin specimen. The power of this technique depends significantly on two parameters: its spatial resolution and the energy resolution available in the spectrum and in the energy-filtered TEM image.

The major use of EFTEM is the mapping of elemental concentrations over large specimen areas with high spatial resolution in the range of $1 \mathrm{~nm}$ and detection sensitivity lower than 1 monolayer $[1,2]$. The procedures for quantitative EFTEM elemental mapping are now very well established and have been successfully applied to a broad range of specimens in materials science and biology [3]. Energy-filtered images can be also used to visualize all physical properties which influence the EELS spectrum, such as electronic properties and bonding states by means of near edge fine structures (ELNES) and of the low-loss part of the spectrum [4].

However these methods have the drawback that spectral information contained in the near edge fine structures (ELNES) and in the low-energy loss region is completely lost. For this reason, energyfiltered image series across the spectral features of the EELS spectrum were developed $[5,6]$ which allowed to double-check the data quality and to extract spatially resolved spectral information from the EFTEM data cube. Recording of a series of EFTEM images with a very narrow slit width allowed to reconstruct ELNES structures and plasmons with an energy resolution estimated to $2 \mathrm{eV}$ and a spatial resolution in the order of $1 \mathrm{~nm}[7,8]$.

Recent developments concentrated on the improvement of EFTEM data acquisition and analysis. With the introduction of new energy-filtering microscopes which are largely corrected for spectral aberrations (Gatan and Zeiss) both transmissivity and non-isochromaticity could be essentially improved [9]. One essential problem of EFTEM is introduced due to sample drift between the exposures. Here, a new automated drift correction method based on digital image filtering, crosscorrelation and statistical evaluation (SDSD) shows better performance than the standard procedures using standard cross-correlation techniques (Fig.1) [10].

Fig.2 shows results from an EFTEM spectrum image of a multilayer system consisting of 4 monolayers of InAs in In(P,As). The original data were recorded with a FEI Tecnai F20, $200 \mathrm{kV}$ field emission TEM with an improved high tension tank, a Wien filter monochromator and a high resolution energy-filter (Gatan) which is corrected for spectral aberrations up to the third order. This instrument allows us to adjust the width of the energy-selecting slit to $0.1 \mathrm{eV}$ with energy steps of $0.1 \mathrm{eV}$. The $\mathrm{P}_{2,3}$ elemental map obtained with the three window method is compared with a $\mathrm{P} \mathrm{L}_{2,3}$ map which was derived from 100 images of the spectrum image. The EFTEM spectrum image was corrected for spatial and energy drift and for non-isochromaticity. The phosphorus distribution is well revealed in the corrected in Fig. 2c. 


\section{References}

[1] F. Hofer et al., Ultramicroscopy 67 (1997) 83.

[2] W. Grogger et al. Ultramicroscopy 96 (2003) 481.

[3] F. Hofer, P. Warbichler, in: Transmission Electron Energy Loss Spectrometry in Materials Science and the EELS Atlas, Ed. C.C. Ahn, Wiley-VCH (2004) 195.

[4] B. Schaffer et al., Micron 34 (2003) 1.

[5] J. Mayer et al., Micron 28 (1997) 361.

[6] P.J. Thomas and P.A. Midgley. Ultramicroscopy 88 (2001) 187.

[7] P. Bayle-Guillemaud et al., J. Microscopy 210 (2003) 66.

[8] W. Sigle et al., Ultramicroscopy 96 (2003) 565.

[9] G. Kothleitner and F. Hofer, Micron 34 (2003) 211.

[10] B. Schaffer, W. Grogger, G. Kothleitner, Ultramicroscopy 102 (2004) 27.

[11] This research was supported by the Special Research Program Electroactive Materials by the Austrian Science Fund (FWF). We would like to thank Martina Dienstleder for FIB crosssectioning and Dr. H. Kirmse (Humboldt University Berlin, Germany) for the multilayer specimen.
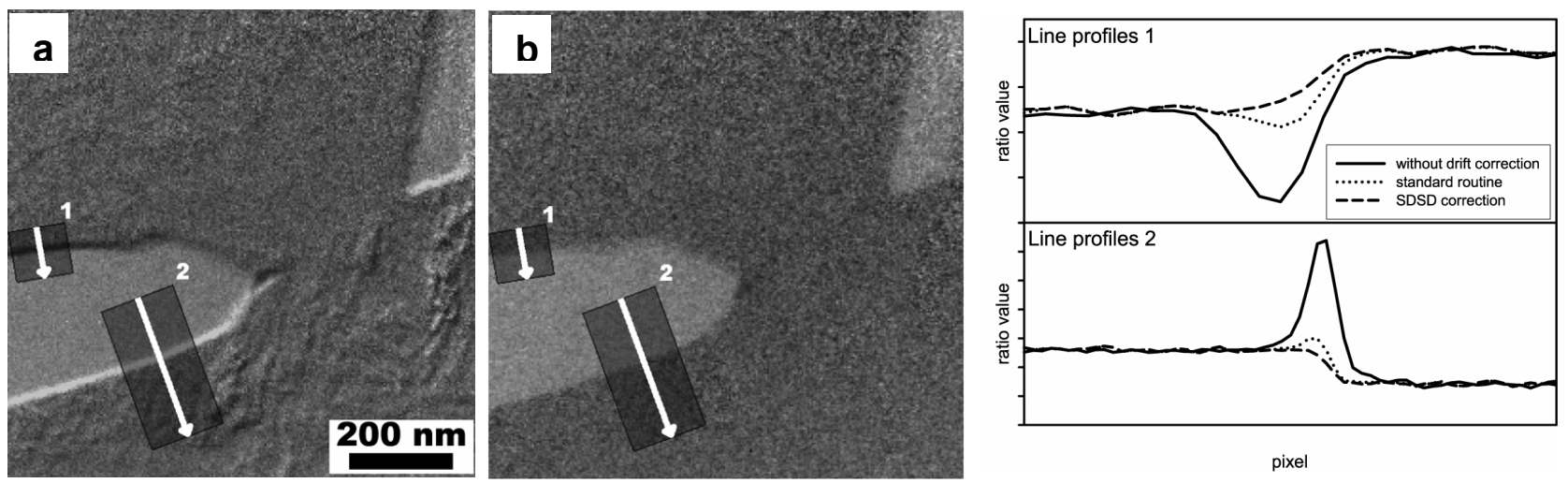

FIG.1. Comparison of ratio images at the $\mathrm{Cr} \mathrm{L}_{2,3}$ edge of precipitates in a steel $(590 \mathrm{eV}$ image divided by $580 \mathrm{eV}$ image) without drift correction (a), with SDSD correction (b); averaged profiles (c) were taken from the indicated areas, Comparison of standard routine and SDSD correction..
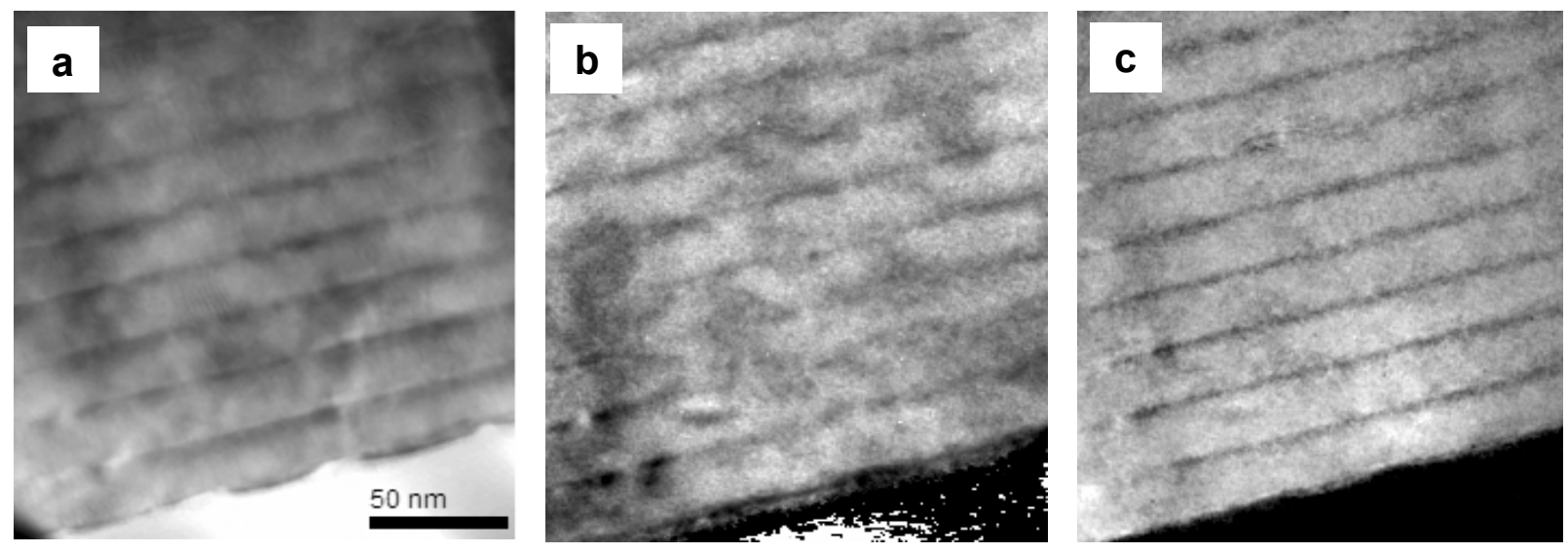

FIG.2. EFTEM spectrum image of an InAs-In(P,As) multilayer sample, (a) TEM image, (b) $\mathrm{P} \mathrm{L}_{2,3}$ elemental map (three window method) and (c) P $\mathrm{L}_{2,3}$ map reconstructed from 100 images. 